\title{
A multigrid method for eigenvalue problems based on shifted-inverse power technique
}

\author{
Hongtao Chen · Yunhui He • Yu Li • Hehu Xie
}

Received: 24 May 2014 / Received: 18 September 2014 / Accepted: 3 November 2014 /

Published online: 7 January 2015

(C) Springer International Publishing AG 2015

\begin{abstract}
A multigrid method is proposed to solve eigenvalue problems by means of the finite element method based on the shifted-inverse power iteration technique. With this scheme, solving eigenvalue problem is transformed to solving a series of nonsingular boundary value problems on multilevel meshes. As replacing the difficult eigenvalue solving by an easier solving of boundary value problems, the multigrid way can improve the overall efficiency of the eigenvalue problem solving. Some numerical experiments are presented to validate the efficiency of this new method.
\end{abstract}

\footnotetext{
This work is supported in part by the National Science Foundation of China (NSFC 91330202, 11001259, 11371026, 11201501, 11301437, 11031006, 11171251, 11201501 2011CB309703), the Natural Science Foundation of Fujian Province of China (2013J05015), the National Basic Research Program (2012CB955804), Tianjin University of Finance and Economics (ZD1302), the National Center for Mathematics and Interdisciplinary Science, CAS and the President Foundation of AMSS-CAS..
}

\section{H. Chen}

School of Mathematical Sciences, Xiamen University, Xiamen 361005, China

e-mail: chenht@xmu.edu.cn

Y. He

LSEC, ICMSEC, Academy of Mathematics and Systems Science, Chinese Academy of Sciences, Beijing 100190, China

e-mail: heyunhui@1sec.cc.ac.cn

Y. Li

Research Center for Mathematics and Economics, Tianjin University of Finance and Economics, Tianjin 300222, China

e-mail: liyu@1sec.cc.ac.cn

H. Xie $(\varangle)$

LSEC, NCMIS, Institute of Computational Mathematics, Academy of Mathematics and Systems Science, Chinese Academy of Sciences, Beijing 100190, China

e-mail: hhxie@1sec.cc.ac.cn 
Keywords Eigenvalue problem - Multigrid - Shifted-inverse power iteration · Finite element method

Mathematics Subject Classification $65 \mathrm{~N} 30 \cdot 65 \mathrm{~N} 25 \cdot 65 \mathrm{~L} 15 \cdot 65 \mathrm{~B} 99$

\section{Introduction}

Solving large scale eigenvalue problems gained a fundamental role in modern science and engineering society. However, it is always a very difficult task to solve highdimensional eigenvalue problems which come from physical and chemical sciences. The papers $[3,7-9,15,22]$ and references therein present some types of multigrid schemes for solving eigenvalue problems which couple the multigrid method with the Rayleigh quotient iteration technique. The involved almost singular linear problems in these methods lead to the numerical unstability. So it is required to design some special solver for these almost singular linear problems $[8,15]$.

The aim of this paper is to present a type of shifted-inverse power iteration method for solving eigenvalue problems based on the multigrid technique. Comparing to the works $[3,7,8,15,22]$, the method presented here is a generalization of the Rayleigh quotient iteration technique and does not need to solve singular linear problems. Recently, we proposed another type of multigrid method $[17,18]$ based on the multilevel correction method [13] which transforms solving eigenvalue problem to solving a series of boundary value problems and solving an eigenvalue problem in a very coarse space. The method proposed here does not need to solve the eigenvalue problem in the coarse space but needs the eigenvalue problem possessing good eigenvalue separations and the initial approximation having good accuracy. The standard Galerkin finite element method for eigenvalue problems has been extensively investigated. See, e.g., Babuška and Osborn [1,2], Chatelin [5] and references therein. Here we adopt some basic results of these papers for our analysis. The corresponding error and computational work of the proposed iteration scheme are analyzed. Based on this analysis, the new method obtains optimal errors with an optimal computational work when we can solve the associated linear problems with the optimal complexity.

In order to describe our method clearly, we give the following simple Laplace eigenvalue problem to illustrate the main idea in this paper, see Sects. 3 and 4 for details: Find $(\lambda, u) \in \mathbb{R} \times H_{0}^{1}(\Omega)$ such that

$$
\begin{aligned}
-\Delta u & =\lambda u \quad & & \text { in } \Omega, \\
u & =0 & & \text { on } \partial \Omega, \\
\int_{\Omega} u^{2} d \Omega & =1, & &
\end{aligned}
$$

where $\Omega \subset \mathbb{R}^{2}$ is a bounded domain with Lipschitz boundary $\partial \Omega$ and $\Delta$ denotes the Laplace operator.

We proceed as follows. First, we construct a series of finite element spaces $V_{h_{1}}, V_{h_{2}}, \ldots, V_{h_{n}} \subset H_{0}^{1}(\Omega)$ defined on the corresponding series of multilevel meshes 
$\mathcal{T}_{h_{k}}, k=1,2, \ldots, n$, such that $V_{h_{1}} \subset V_{h_{2}} \subset \cdots \subset V_{h_{n}}$ and $h_{k}=h_{k-1} / \beta[4,6]$. Our multigrid algorithm for the approximation of the eigenpairs is

1. Solve an eigenvalue problem in the coarsest space $V_{h_{1}}$ : Find $\left(\lambda_{h_{1}}, u_{h_{1}}\right) \in \mathbb{R} \times V_{h_{1}}$ such that $\left\|\nabla u_{h_{1}}\right\|_{0}=1$ and

$$
\int_{\Omega} \nabla u_{h_{1}} \nabla v_{h_{1}} d \Omega=\lambda_{h_{1}} \int_{\Omega} u_{h_{1}} v_{h_{1}} d \Omega, \quad v_{h_{1}} \in V_{h_{1}} .
$$

2. Do $k=1, \ldots, n-1$

- Solve the following auxiliary boundary value problem: Find $\widetilde{u}_{h_{k+1}} \in V_{h_{k+1}}$ such that for any $v_{h_{k+1}} \in V_{h_{k+1}}$,

$$
\int_{\Omega}\left(\nabla \widetilde{u}_{h_{k+1}} \nabla v_{h_{k+1}}-\alpha_{k+1} \widetilde{u}_{h_{k+1}} v_{h_{k+1}}\right) d \Omega=\int_{\Omega} u_{h_{k}} v_{h_{k+1}} d \Omega .
$$

- Do the normalization

$$
u_{h_{k+1}}=\frac{\widetilde{u}_{h_{k+1}}}{\left\|\nabla \widetilde{u}_{h_{k+1}}\right\|_{0}}
$$

and compute the Rayleigh quotient for $u_{h_{k+1}}$,

$$
\lambda_{h_{k+1}}=\frac{\left\|\nabla u_{h_{k+1}}\right\|_{0}^{2}}{\left\|u_{h_{k+1}}\right\|_{0}^{2}} .
$$

\section{End Do}

If, for example, $\lambda_{h_{1}}$ is the approximation for the first eigenvalue of problem (1) at the first step and $\Omega$ is a convex domain, then we can establish the following results by taking a suitable choice of $\alpha_{k+1}$, see Sects. 3 and 4 for details:

$$
\left\|\nabla\left(u-u_{h_{n}}\right)\right\|_{0}=\mathcal{O}\left(h_{n}\right) \quad \text { and } \quad\left|\lambda-\lambda_{h_{n}}\right|=\mathcal{O}\left(h_{n}^{2}\right) .
$$

These two estimates mean that we obtain asymptotically optimal errors.

In this method, we replace solving eigenvalue problem on the finest finite element spaces by solving a series of boundary value problems in the corresponding series of finite element spaces and an eigenvalue problem in the initial finite element space.

An outline of the paper goes as follows. In Sect. 2, we introduce the finite element method for the eigenvalue problem and give the corresponding basic error estimates. A type of one shifted-inverse power iteration step is given in Sect. 3. In Sect. 4, we propose a type of multigrid algorithm for the eigenvalue problem based on the shifted-inverse power iteration step. The computational work estimate of the eigenvalue multigrid method is given in Sect. 5. In Sect. 6, two numerical examples are presented to validate our theoretical analysis. Some concluding remarks are given in the last section. 


\section{Discretization by finite element method}

In this section, we introduce some notation and error estimates of the finite element approximation for the eigenvalue problem. The letter $C$ (with or without subscripts) denotes a generic positive constant which may be different at its different occurrences through the paper. Notation $x_{1} \lesssim y_{1}, x_{2} \gtrsim y_{2}$ and $x_{3} \approx y_{3}$ mean that $x_{1} \leq C_{1} y_{1}$, $x_{2} \geq c_{2} y_{2}$ and $c_{3} x_{3} \leq y_{3} \leq C_{3} x_{3}$ for some constants $C_{1}, c_{2}, c_{3}$ and $C_{3}$ that are independent of mesh sizes [19].

Let $(V,\|\cdot\|)$ be a real Hilbert space with the inner product $(\cdot, \cdot)$ and norm $\|\cdot\|$, respectively. Let $a(\cdot, \cdot)$ and $b(\cdot, \cdot)$ be two symmetric bilinear forms on $V \times V$ satisfying

$$
\begin{aligned}
a(w, v) & \lesssim\|w\|\|v\|, & & w, v \in V, \\
\|w\|^{2} & \lesssim a(w, w), & & w \in V, \\
0 & <b(w, w), & & 0 \neq w \in V .
\end{aligned}
$$

From (2) and (3), we know that $\|\cdot\|_{a}=a(\cdot, \cdot)^{1 / 2}$ and $\|\cdot\|$ are two equivalent norms on $V$. Assume that the norm $\|\cdot\|$ is relatively compact with respect to the norm $\|\cdot\|_{b}=$ $b(\cdot, \cdot)^{1 / 2}$ in the sense that from any sequence bounded in $\|\cdot\|$ one can extract a subsequence which is Cauchy with respect to $\|\cdot\|_{b}$. In what follows we shall use $a(\cdot, \cdot)$ and $\|\cdot\|_{a}$, respectively, as the inner product and norm on $V$.

We assume that $V_{h} \subset V$ is a family of finite-dimensional spaces that satisfy the following assumption: For any $w \in V$,

$$
\lim _{h \rightarrow 0} \inf _{v_{h} \in V_{h}}\left\|w-v_{h}\right\|_{a}=0 .
$$

Let $P_{h}$ be the finite element projection operator of $V$ onto $V_{h}$ defined by

$$
a\left(w-P_{h} w, v_{h}\right)=0, \quad w \in V, \quad v_{h} \in V_{h} .
$$

Obviously, $\left\|P_{h} w\right\|_{a} \leq\|w\|_{a}$ for all $w \in V$. For any $w \in V$, by (4) we have

$$
\left\|w-P_{h} w\right\|_{a}=o(1) \quad \text { as } h \rightarrow 0 .
$$

Define $\eta_{a}(h)$ as

$$
\eta_{a}(h)=\sup _{\substack{f \in V^{\prime} \\\|f\|_{b}=1}} \inf _{v_{h} \in V_{h}}\left\|T f-v_{h}\right\|_{a},
$$

where $V^{\prime}$ denotes the dual space of $V$ and the operator $T: V^{\prime} \rightarrow V$ is defined as

$$
a(T f, v)=b(f, v), \quad f \in V^{\prime}, \quad v \in V .
$$


In order to derive the error estimate of eigenpair approximation in the weak norm $\|\cdot\|_{b}$, we need the following weak norm error estimates of the finite element projection operator $P_{h}$ [2, Lemmas $\left.3.3 \& 3.4\right]$ :

$$
\eta_{a}(h) \rightarrow 0 \quad \text { as } h \rightarrow 0
$$

and

$$
\left\|w-P_{h} w\right\|_{b} \lesssim \eta_{a}(h)\left\|w-P_{h} w\right\|_{a}, \quad w \in V
$$

In our methodology description, we are concerned with the following general eigenvalue problem: Find $(\lambda, u) \in \mathbb{R} \times V$ such that $a(u, u)=1$ and

$$
a(u, v)=\lambda b(u, v), \quad v \in V .
$$

Under the assumptions we have made, (5) has an eigenvalue sequence $\left\{\lambda_{j}\right\}[2,5]$,

$$
0<\lambda_{1} \leq \lambda_{2} \leq \cdots \leq \lambda_{k} \leq \cdots, \quad \lim _{k \rightarrow \infty} \lambda_{k}=\infty
$$

and the associated eigenfunctions

$$
u_{1}, u_{2}, \ldots, u_{k}, \ldots
$$

with $b\left(u_{i}, u_{j}\right)=\delta_{i j}$. In the sequence $\left\{\lambda_{j}\right\}, \lambda_{j}$ are repeated according to their geometric multiplicity.

For the eigenvalue $\lambda$, there exists the following Rayleigh quotient expression $[1,2$, 21]:

$$
\lambda=\frac{a(u, u)}{b(u, u)} .
$$

Let $M\left(\lambda_{i}\right)$ denote the eigenfunction space corresponding to the eigenvalue $\lambda_{i}$ which is defined by

$$
M\left(\lambda_{i}\right)=\left\{w \in V: w \text { is an eigenfunction of (5) corresponding to } \lambda_{i}\right\} .
$$

Then $[1,2]$

$$
\lambda_{i}=\inf _{\substack{v \in V \\ v \perp M\left(\lambda_{j}\right) \\ \lambda_{j}<\lambda_{i}}} \frac{a(v, v)}{b(v, v)} .
$$

Now, let us define the finite element approximations of problem (5). First we generate a shape-regular decomposition of the computing domain $\Omega \subset \mathbb{R}^{d}, d=2,3$, into triangles or rectangles for $d=2$ (tetrahedrons or hexahedrons for $d=3$ ). The diameter of a cell $K \in \mathcal{T}_{h}$ is denoted by $h_{K}$. The mesh diameter $h$ describes the 
maximum diameter of all cells $K \in \mathcal{T}_{h}$. Based on the mesh $\mathcal{T}_{h}$, we can construct a finite element space denoted by $V_{h} \subset V$.

Then we define the approximation for the eigenpair $(\lambda, u)$ of (5) by the finite element method as: Find $\left(\bar{\lambda}_{h}, \bar{u}_{h}\right) \in \mathbb{R} \times V_{h}$ such that $a\left(\bar{u}_{h}, \bar{u}_{h}\right)=1$ and

$$
a\left(\bar{u}_{h}, v_{h}\right)=\bar{\lambda}_{h} b\left(\bar{u}_{h}, v_{h}\right), \quad v_{h} \in V_{h} .
$$

Again under the assumptions we have made, (6) has the eigenvalues [2,5]

$$
0<\bar{\lambda}_{1, h} \leq \bar{\lambda}_{2, h} \leq \cdots \leq \bar{\lambda}_{k, h} \leq \cdots \leq \bar{\lambda}_{N_{h}, h}
$$

and the corresponding eigenfunctions

$$
\bar{u}_{1, h}, \quad \bar{u}_{2, h}, \quad \ldots, \quad \bar{u}_{k, h}, \quad \ldots, \quad \bar{u}_{N_{h}, h}
$$

with $b\left(\bar{u}_{i, h}, \bar{u}_{j, h}\right)=\delta_{i j}, 1 \leq i, j \leq N_{h}\left(N_{h}\right.$ is the dimension of the finite element space $\left.V_{h}\right)$.

The following Rayleigh quotient expression for $\bar{\lambda}_{h}$ holds $[1,2,21]$ :

$$
\bar{\lambda}_{h}=\frac{a\left(\bar{u}_{h}, \bar{u}_{h}\right)}{b\left(\bar{u}_{h}, \bar{u}_{h}\right)},
$$

From the minimum-maximum principle $[1,2]$, the following upper bound result holds:

$$
\lambda_{i} \leq \bar{\lambda}_{i, h}, \quad i=1,2, \ldots, N_{h}
$$

Similarly, let $M_{h}\left(\lambda_{i}\right)$ denote the approximate eigenfunction space corresponding to the eigenvalue $\lambda_{i}$ which is defined by

$$
M_{h}\left(\lambda_{i}\right)=\left\{w_{h} \in V_{h}: w_{h} \text { is an eigenfunction of (6) corresponding to } \lambda_{i}\right\} .
$$

From [1,2], each eigenvalue $\lambda_{i, h}$ can be defined as follows:

$$
\lambda_{i, h}=\inf _{\substack{v_{h} \in V_{h} \\ v h \perp M_{h}\left(\lambda_{j}\right) \\ \lambda_{j}<\lambda_{i}}} \frac{a\left(v_{h}, v_{h}\right)}{b\left(v_{h}, v_{h}\right)}
$$

Then we define

$$
\delta_{h}\left(\lambda_{i}\right)=\sup _{\substack{w \in M\left(\lambda_{i}\right) \\\|w\|_{a}=1}} \inf _{v_{h} \in V_{h}}\left\|w-v_{h}\right\|_{a}
$$

For the analysis in this paper, we introduce the spectral projection [2],

$$
\Pi_{1, h}=\frac{1}{2 \pi \mathrm{i}} \int_{\Gamma}\left(z-P_{h} T\right)^{-1} d z,
$$


where $\Gamma$ is a Jordan curve in $\mathbb{C}$ that encloses the eigenvalue $\lambda_{1}$ and no other eigenvalues.

For the eigenpair approximations by the finite element method, there exist the following error estimates.

Proposition 2.1 [1, Lemma 3.7, (3. 29b)], [2, p. 699], [5]

- For any eigenfunction approximation $\bar{u}_{i, h}$ of (6), $i=1,2, \ldots, N_{h}$, there is an eigenfunction $u_{i}$ of (5) corresponding to $\lambda_{i}$ such that $\left\|u_{i}\right\|_{a}=1$ and

$$
\left\|u_{i}-\bar{u}_{i, h}\right\|_{a} \leq C \delta_{h}\left(\lambda_{i}\right)
$$

Furthermore,

$$
\left\|u_{i}-\bar{u}_{i, h}\right\|_{b} \leq C \eta_{a}(h)\left\|u_{i}-\bar{u}_{i, h}\right\|_{a} .
$$

- For each eigenvalue, we have

$$
\lambda_{i} \leq \bar{\lambda}_{i, h} \leq \lambda_{i}+C \delta_{h}^{2}\left(\lambda_{i}\right)
$$

Here and hereafter $C$ is some constant depending on $\lambda_{i}$ but independent of the mesh size $h$.

\section{One shifted-inverse power iteration step with multigrid method}

In this section, we present a type of one shifted-inverse power iteration step to improve the accuracy of the given eigenvalue and eigenfunction approximations. This iteration method requires solving only auxiliary boundary value problems in the finer finite element space.

To analyze our method, we introduce the following error expansion of the eigenvalue by the Rayleigh quotient formula.

Lemma $3.1[1,2,14,21]$ Assume $(\lambda, u)$ is a true solution of the eigenvalue problem (5) and $0 \neq \psi \in V$. Let us define

$$
\widehat{\lambda}=\frac{a(\psi, \psi)}{b(\psi, \psi)} .
$$

Then

$$
\widehat{\lambda}-\lambda=\frac{a(u-\psi, u-\psi)}{b(\psi, \psi)}-\lambda \frac{b(u-\psi, u-\psi)}{b(\psi, \psi)} .
$$

For simplicity, here we state only the numerical method for the first eigenvalue $\lambda_{1}$. Assume we have obtained an eigenpair approximation $\left(\lambda_{1, h_{k}}, u_{1, h_{k}}\right) \in \mathbb{R} \times V_{h_{k}}$ with $\left\|u_{1, h_{k}}\right\|_{a}=1$. Now we introduce a type of iteration step to improve the accuracy of the current eigenpair approximation $\left(\lambda_{1, h_{k}}, u_{1, h_{k}}\right)$. Let $V_{h_{k+1}} \subset V$ be a finer finite element space such that $V_{h_{k}} \subset V_{h_{k+1}}$. Based on this finer finite element space, we define the following one shifted-inverse power iteration step. 


\section{Algorithm 3.2 One SHIFTED- INVERSE POWER ITERATION STEP}

1. Solve the following boundary value problem: Find $\widehat{u}_{1, h_{k+1}} \in V_{h_{k+1}}$ such that for any $v_{h_{k+1}} \in V_{h_{k+1}}$,

$$
a\left(\widehat{u}_{1, h_{k+1}}, v_{h_{k+1}}\right)-\alpha_{1, k+1} b\left(\widehat{u}_{1, h_{k+1}}, v_{h_{k+1}}\right)=b\left(u_{1, h_{k}}, v_{h_{k+1}}\right) .
$$

2. Do the normalization for $\widehat{u}_{1, h_{k+1}}$,

$$
u_{1, h_{k+1}}=\frac{\widehat{u}_{1, h_{k+1}}}{\left\|\widehat{u}_{1, h_{k+1}}\right\|_{a}}
$$

and compute the Rayleigh quotient for $u_{1, h_{k+1}}$,

$$
\lambda_{1, h_{k+1}}=\frac{a\left(u_{1, h_{k+1}}, u_{1, h_{k+1}}\right)}{b\left(u_{1, h_{k+1}}, u_{1, h_{k+1}}\right)} .
$$

Then we obtain a new eigenpair approximation $\left(\lambda_{1, h_{k+1}}, u_{1, h_{k+1}}\right) \in \mathbb{R} \times V_{h_{k+1}}$. Summarize the above two steps into

$$
\left(\lambda_{1, h_{k+1}}, u_{1, h_{k+1}}\right)=\operatorname{Correction}\left(\alpha_{1, k+1}, u_{1, h_{k}}, V_{h_{k+1}}\right) .
$$

Theorem 3.3 When $\alpha_{1, k+1} \neq \bar{\lambda}_{1, h_{k+1}}$ and $\alpha_{1, k+1}<\bar{\lambda}_{2, h_{k+1}}$, after one correction step, the resultant approximation $u_{1, h_{k+1}} \in V_{h_{k+1}}$ has the following error estimate:

$$
\left\|u_{1, h_{k+1}}-\Pi_{1, h_{k+1}} u_{1, h_{k+1}}\right\|_{a} \leq \frac{\theta_{1, k+1}\left\|u_{1, h_{k}}-\Pi_{1, h_{k+1}} u_{1, h_{k}}\right\|_{a}}{1-\left(1+\theta_{1, k+1}\right)\left\|u_{1, h_{k}}-\Pi_{1, h_{k+1}} u_{1, h_{k}}\right\|_{a}},
$$

where

$$
\theta_{1, k+1}=\frac{\left|\bar{\lambda}_{1, h_{k+1}}-\alpha_{1, k+1}\right|}{\bar{\lambda}_{2, h_{k+1}}-\alpha_{1, k+1}} .
$$

Proof Let us define $\gamma=\bar{\lambda}_{1, h_{k+1}}-\alpha_{1, k+1}, \widetilde{u}_{1, h_{k+1}}=\gamma \widehat{u}_{1, h_{k+1}}$ and $w_{h_{k+1}}=\widetilde{u}_{1, h_{k+1}}-$ $\Pi_{1, h_{k+1}} u_{1, h_{k}}$. Then for any $v_{h_{k+1}} \in V_{h_{k+1}}$,

$$
a\left(\widetilde{u}_{1, h_{k+1}}, v_{h_{k+1}}\right)-\alpha_{1, k+1} b\left(\widetilde{u}_{1, h_{k+1}}, v_{h_{k+1}}\right)=\gamma b\left(u_{1, h_{k}}, v_{h_{k+1}}\right) .
$$

From $u_{1, h_{k}}-\Pi_{1, h_{k+1}} u_{1, h_{k}} \perp M_{h_{k+1}}\left(\lambda_{1}\right), \Pi_{1, h_{k+1}} u_{1, h_{k}} \in M_{h_{k+1}}\left(\lambda_{1}\right),(13)$ and for any $v_{h_{k+1}} \in V_{h_{k+1}}$,

$$
\begin{aligned}
a\left(\Pi_{1, h_{k+1}} u_{1, h_{k}}, v_{h_{k+1}}\right)-\alpha_{1, k+1} b\left(\Pi_{1, h_{k+1}} u_{1, h_{k}}, v_{h_{k+1}}\right) & \\
& =\gamma b\left(\Pi_{1, h_{k+1}} u_{1, h_{k}}, v_{h_{k+1}}\right)
\end{aligned}
$$

we have $w_{h_{k+1}} \perp M_{h_{k+1}}\left(\lambda_{1}\right)$ and $a\left(w_{h_{k+1}}, \Pi_{1, h_{k+1}} u_{1, h_{k}}\right)=0$. 
From (7), (13) and (14), the following estimate holds:

$$
\begin{aligned}
\left\|w_{h_{k+1}}\right\|_{a}^{2} & =\alpha_{1, k+1} b\left(w_{h_{k+1}}, w_{h_{k+1}}\right)+\gamma b\left(u_{1, h_{k}}-\Pi_{1, h_{k+1}} u_{1, h_{k}}, w_{h_{k+1}}\right) \\
& \leq \frac{\alpha_{1, k+1}}{\bar{\lambda}_{2, h_{k+1}}}\left\|w_{h_{k+1}}\right\|_{a}^{2}+|\gamma|\left\|u_{1, h_{k}}-\Pi_{1, h_{k+1}} u_{1, h_{k}}\right\|_{b}\left\|w_{h_{k+1}}\right\|_{b} \\
& \leq \frac{\alpha_{1, k+1}}{\bar{\lambda}_{2, h_{k+1}}}\left\|w_{h_{k+1}}\right\|_{a}^{2}+\frac{|\gamma|}{\bar{\lambda}_{2, h_{k+1}}}\left\|u_{1, h_{k}}-\Pi_{1, h_{k+1}} u_{1, h_{k}}\right\|_{a}\left\|w_{h_{k+1}}\right\|_{a} .
\end{aligned}
$$

Then we have

$$
\left\|w_{h_{k+1}}\right\|_{a} \leq \frac{\left|\bar{\lambda}_{1, h_{k+1}}-\alpha_{1, k+1}\right|}{\bar{\lambda}_{2, h_{k+1}}-\alpha_{1, k+1}}\left\|u_{1, h_{k}}-\Pi_{1, h_{k+1}} u_{1, h_{k}}\right\|_{a} .
$$

From (15), $\left\|u_{1, h_{k}}\right\|_{a}=1$ and $\widetilde{u}_{1, h_{k+1}}=u_{1, h_{k}}+\Pi_{1, h_{k+1}} u_{1, h_{k}}-u_{1, h_{k}}+\widetilde{u}_{1, h_{k+1}}-$ $\Pi_{1, h_{k+1}} u_{1, h_{k}}$, the following estimate holds:

$$
\begin{aligned}
\left\|\tilde{u}_{1, h_{k+1}}\right\|_{a} & \geq\left\|u_{1, h_{k}}\right\|_{a}-\left\|\Pi_{1, h_{k+1}} u_{1, h_{k}}-u_{1, h_{k}}\right\|_{a}-\left\|\tilde{u}_{1, h_{k+1}}-\Pi_{1, h_{k+1}} u_{1, h_{k}}\right\|_{a} \\
& \geq 1-\left(1+\theta_{1, k+1}\right)\left\|u_{1, h_{k}}-\Pi_{1, h_{k+1}} u_{1, h_{k}}\right\|_{a} .
\end{aligned}
$$

Whence

$$
\frac{1}{\left\|\tilde{u}_{1, h_{k+1}}\right\|_{a}} \leq \frac{1}{1-\left(1+\theta_{1, k+1}\right)\left\|u_{1, h_{k}}-\Pi_{1, h_{k+1}} u_{1, h_{k}}\right\|_{a}}
$$

Since $u_{1, h_{k+1}}=\tilde{u}_{1, h_{k+1}} /\left\|\tilde{u}_{1, h_{k+1}}\right\|_{a}$ and $w_{h_{k+1}} \perp M_{h_{k+1}}\left(\lambda_{1}\right)$, the following estimate holds:

$$
\begin{aligned}
\left\|u_{1, h_{k+1}}-\Pi_{1, h_{k+1}} u_{1, h_{k+1}}\right\|_{a} & =\frac{\left\|\tilde{u}_{1, h_{k+1}}-\Pi_{1, h_{k+1}} u_{1, h_{k}}\right\|_{a}}{\left\|\tilde{u}_{1, h_{k+1}}\right\|_{a}} \\
& \leq \frac{\theta_{1, k+1}\left\|u_{1, h_{k}}-\Pi_{1, h_{k+1}} u_{1, h_{k}}\right\|_{a}}{1-\left(1+\theta_{1, k+1}\right)\left\|u_{1, h_{k}}-\Pi_{1, h_{k+1}} u_{1, h_{k}}\right\|_{a}},
\end{aligned}
$$

which means we have obtained the desired result (11) and the proof is complete.

Remark 3.4 Let us discuss two choices of $\alpha_{1, k+1}$.

- If $\alpha_{1, k+1}=0$, i.e. we do not use a shift, then Algorithm 3.2 just repeats the well-known two grid method [21]. From (11), we have

$$
\left\|u_{1, h_{k+1}}-\Pi_{1, h_{k+1}} u_{1, h_{k+1}}\right\|_{a} \leq \kappa_{1, k+1}\left\|u_{1, h_{k}}-\Pi_{1, h_{k+1}} u_{1, h_{k}}\right\|_{a},
$$

where

$$
\kappa_{1, k+1}=\frac{\bar{\lambda}_{1, h_{k+1}} / \bar{\lambda}_{2, h_{k+1}}}{1-\left(1+\bar{\lambda}_{1, h_{k+1}} / \bar{\lambda}_{2, h_{k+1}}\right)\left\|u_{1, h_{k}}-\Pi_{1, h_{k+1}} u_{1, h_{k}}\right\|_{a}} .
$$


This indicates that the two grid step has linear convergence speed rate when $\| u_{1, h_{k}}-$ $\Pi_{1, h_{k+1}} u_{1, h_{k}} \|_{a}$ is small enough. But if the gap between $\bar{\lambda}_{1, h_{k+1}}$ and $\bar{\lambda}_{2, h_{k+1}}$ is small, the convergence is slow.

- From Lemma 3.1, the following estimate holds:

$$
\begin{aligned}
\lambda_{1, h_{k}}-\bar{\lambda}_{1, h_{k+1}}= & \frac{a\left(\Pi_{1, h_{k+1}} u_{1, h_{k}}-u_{1, h_{k}}, \Pi_{1, h_{k+1}} u_{1, h_{k}}-u_{1, h_{k}}\right)}{b\left(u_{1, h_{k}}, u_{1, h_{k}}\right)} \\
& -\bar{\lambda}_{1, h_{k+1}} \frac{b\left(\Pi_{1, h_{k+1}} u_{1, h_{k}}-u_{1, h_{k}}, \Pi_{1, h_{k+1}} u_{1, h_{k}}-u_{1, h_{k}}\right)}{b\left(u_{1, h_{k}}, u_{1, h_{k}}\right)} \\
& \lesssim\left\|u_{1, h_{k}}-\Pi_{1, h_{k+1}} u_{1, h_{k}}\right\|_{a}^{2} .
\end{aligned}
$$

If $\alpha_{1, h_{k+1}}=\lambda_{1, h_{k}}$, from (11), we have

$$
\left\|u_{1, h_{k+1}}-\Pi_{1, h_{k+1}} u_{1, h_{k+1}}\right\|_{a} \lesssim\left\|u_{1, h_{k}}-\Pi_{1, h_{k+1}} u_{1, h_{k}}\right\|_{a}^{3},
$$

which means that the Rayleigh quotient iteration has cubic convergence rate.

The suitable choice for $\alpha_{1, k+1}$ sometimes is not so easy to obtain since it depends on $\bar{\lambda}_{1, h_{k+1}}$ and $\bar{\lambda}_{2, h_{k+1}}$ which are unknown. But from (11), if

$$
0<\frac{\left|\bar{\lambda}_{1, h_{k+1}}-\alpha_{1, k+1}\right|}{\bar{\lambda}_{2, h_{k+1}}-\alpha_{1, k+1}}<1
$$

then the accuracy for the solution of (10) can be improved through more iterations. Then we can design the following modified one multi shifted-inverse power iteration step.

\section{Algorithm 3.5 Multi ShIFTED- INVERSE POWer Iteration STEP}

1. Set $u_{1, h_{k+1}}^{0}=u_{1, h_{k}}$.

2. Do $j=0, \ldots, \ell-1$

- Solve the following boundary value problem: Find $\widehat{u}_{1, h_{k+1}}^{j+1} \in V_{h_{k+1}}$ such that for any $v_{h_{k+1}} \in V_{h_{k+1}}$,

$$
a\left(\widehat{u}_{1, h_{k+1}}^{j+1}, v_{h_{k+1}}\right)-\alpha_{1, k+1}^{j+1} b\left(\widehat{u}_{1, h_{k+1}}^{j+1}, v_{h_{k+1}}\right)=b\left(u_{1, h_{k+1}}^{j}, v_{h_{k+1}}\right) .
$$

- Normalize $\widehat{u}_{1, h_{k+1}}^{j+1}$ by

$$
u_{1, h_{k+1}}^{j+1}=\frac{\widehat{u}_{1, h_{k+1}}^{j+1}}{\left\|\widehat{u}_{1, h_{k+1}}^{j+1}\right\|_{a}}
$$


and compute the Rayleigh quotient for $u_{1, h_{k+1}}^{j+1}$,

$$
\lambda_{1, h_{k+1}}^{j+1}=\frac{a\left(u_{1, h_{k+1}}^{j+1}, u_{1, h_{k+1}}^{j+1}\right)}{b\left(u_{1, h_{k+1}}^{j+1}, u_{1, h_{k+1}}^{j+1}\right)} .
$$

\section{End Do}

3. Set $u_{1, h_{k+1}}=u_{1, h_{k+1}}^{\ell}$ and $\lambda_{1, h_{k+1}}=\lambda_{1, h_{k+1}}^{\ell}$.

Then we obtain a new eigenpair approximation $\left(\lambda_{1, h_{k+1}}, u_{1, h_{k+1}}\right) \in \mathbb{R} \times V_{h_{k+1}}$. Summarize the above two steps into

$$
\left(\lambda_{1, h_{k+1}}, u_{1, h_{k+1}}\right)=\operatorname{Correction}\left(\left\{\alpha_{1, k+1}^{j}\right\}_{j=1}^{\ell}, u_{1, h_{k}}, V_{h_{k+1}}\right) .
$$

In Algorithm 3.5, we can adjust $\ell$ so that the following estimate holds:

$$
\left\|u_{1, h_{k+1}}-\Pi_{1, h_{k+1}} u_{1, h_{k+1}}\right\|_{a}<\frac{1}{\beta}\left\|u_{1, h_{k}}-\Pi_{1, h_{k+1}} u_{1, h_{k}}\right\|_{a},
$$

where $\beta$ is a constant defined in the following section. In fact, $\ell$ can be very small ( $\ell=2$ or 3 ) and the modified iteration step makes the choice of $\alpha_{1, k+1}$ become not so sharp as in Algorithm 3.2 what improves the stability of the iteration step.

\section{Multigrid scheme for the eigenvalue problem}

In this section, we introduce a type of multigrid scheme based on ONE SHIFTEDINVERSE POWER ITERATION STEP defined in Algorithm 3.2 or 3.5. This type of multigrid method obtains the same optimal error estimate as solving the eigenvalue problem directly in the finest finite element space.

In order to do the multigrid scheme, we define a sequence of triangulations $\mathcal{T}_{h_{k}}$ of $\Omega$ determined as follows. Suppose $\mathcal{T}_{h_{1}}$ is given and let $\mathcal{T}_{h_{k}}$ be obtained from $\mathcal{T}_{h_{k-1}}$ via regular refinement (produce $\beta^{d}$ subelements) such that

$$
h_{k}=\frac{1}{\beta} h_{k-1} \text {. }
$$

Based on this sequence of meshes, we construct the corresponding linear finite element spaces such that

$$
V_{h_{1}} \subset V_{h_{2}} \subset \cdots \subset V_{h_{n}}
$$

the the following relations of approximation errors hold:

$$
\eta_{a}\left(h_{k}\right) \approx \frac{1}{\beta} \eta_{a}\left(h_{k-1}\right), \quad \delta_{h_{k}}(\lambda) \approx \frac{1}{\beta} \delta_{h_{k-1}}(\lambda), \quad k=2, \ldots, n .
$$

From the spectral projection definition (8), we have

$$
\left\|\Pi_{1, h_{k}} u_{1, h_{k}}-\Pi_{1, h_{k+1}} u_{1, h_{k}}\right\|_{a} \leq C_{4} \delta_{h_{k}}\left(\lambda_{1}\right), \quad k=1, \ldots, n-1,
$$


where the constant $C_{4}$ is independent of the mesh size $h_{k}$.

\section{Algorithm 4.1 Eigenvalue Multigrid Scheme}

1. Construct a series of nested finite element spaces $V_{h_{1}}, V_{h_{2}}, \ldots, V_{h_{n}}$ such that (16) and (17) hold.

2. Solve the following eigenvalue problem: Find $\left(\lambda_{1, h_{1}}, u_{1, h_{1}}\right) \in \mathbb{R} \times V_{h_{1}}$ such that $a\left(u_{1, h_{1}}, u_{1, h_{1}}\right)=1$ and

$$
a\left(u_{1, h_{1}}, v_{h_{1}}\right)=\lambda_{1, h_{1}} b\left(u_{1, h_{1}}, v_{h_{1}}\right), \quad v_{h_{1}} \in V_{h_{1}} .
$$

3. Do $k=1, \ldots, n-1$

Obtain a new eigenpair approximation $\left(\lambda_{1, h_{k+1}}, u_{1, h_{k+1}}\right) \in \mathbb{R} \times V_{h_{k+1}}$ by a correction step

$$
\left(\lambda_{1, h_{k+1}}, u_{1, h_{k+1}}\right)=\operatorname{Correction}\left(\alpha_{1, k+1}, u_{1, h_{k}}, V_{h_{k+1}}\right) .
$$

\section{End Do}

Finally, we obtain an eigenpair approximation $\left(\lambda_{1, h_{n}}, u_{1, h_{n}}\right) \in \mathbb{R} \times V_{h_{n}}$.

For simplicity in this paper, we assume the following estimates hold:

$$
\eta_{a}\left(h_{k}\right) \approx h_{k}, \quad \delta_{h_{k}}\left(\lambda_{1}\right) \approx h_{k}, \quad k=1, \ldots, n
$$

Theorem 4.2 After implementing Algorithm 4.1, the resultant eigenpair approximation $\left(\lambda_{1, h_{n}}, u_{1, h_{n}}\right)$ has the following error estimates:

$$
\begin{aligned}
\left\|u_{1, h_{n}}-\Pi_{1, h_{n}} u_{1, h_{n}}\right\|_{a} & \leq C_{5} \delta_{h_{n}}\left(\lambda_{1}\right), \\
\left|\lambda_{1, h_{n}}-\bar{\lambda}_{1, h_{n}}\right| & \leq C_{5} \delta_{h_{n}}^{2}\left(\lambda_{1}\right),
\end{aligned}
$$

when the mesh size $h_{1}$ is small enough and $\alpha_{1, k+1}$ is chosen as follows:

$$
\alpha_{1, k+1}=\max \left\{0, \frac{2\left(1+C_{4} / C_{5}\right) \beta \lambda_{1, h_{k}}-\lambda_{2, h_{1}}}{2\left(1+C_{4} / C_{5}\right) \beta-1}\right\}, \quad k=1, \ldots, n-1,
$$

where $C_{5}$ is a constant not less than the constant $C$ in (9).

Then there exists a constant $C_{6}$ such that the following final convergence results hold:

$$
\left\|u_{1}-u_{1, h_{n}}\right\|_{a} \leq C_{6} \delta_{h_{n}}\left(\lambda_{1}\right), \quad\left|\lambda_{1}-\lambda_{1, h_{n}}\right| \leq C_{6} \delta_{h_{n}}^{2}\left(\lambda_{1}\right)
$$

Proof First, it is obvious that $2\left(1+C_{4} / C_{5}\right) \beta>1$. If we choose $\alpha_{1, k+1}$ as in (21) and $2\left(1+C_{4} / C_{5}\right) \beta \lambda_{1, h_{k}}-\lambda_{2, h_{1}}>0$, then

$$
\alpha_{1, k+1}=\frac{2\left(1+C_{4} / C_{5}\right) \beta \lambda_{1, h_{k}}-\lambda_{2, h_{1}}}{2\left(1+C_{4} / C_{5}\right) \beta-1}
$$


and the following estimate holds:

$$
\begin{aligned}
\theta_{1, k+1} & =\frac{\left|\bar{\lambda}_{1, h_{k+1}}-\alpha_{1, k+1}\right|}{\bar{\lambda}_{2, h_{k+1}}-\alpha_{1, k+1}} \\
& =\frac{\left|\lambda_{2, h_{1}}-\bar{\lambda}_{1, h_{k+1}}+2\left(1+C_{4} / C_{5}\right) \beta\left(\bar{\lambda}_{1, h_{k+1}}-\lambda_{1, h_{k}}\right)\right|}{2\left(1+C_{4} / C_{5}\right) \beta\left(\bar{\lambda}_{2, h_{k+1}}-\lambda_{1, h_{k}}\right)+\lambda_{2, h_{1}}-\bar{\lambda}_{2, h_{k+1}}} \\
& =\frac{1}{2\left(1+C_{4} / C_{5}\right) \beta}+\mathcal{O}\left(h_{1}^{2}\right) .
\end{aligned}
$$

If we choose $\alpha_{1, k+1}$ as in (21) and $2\left(1+C_{4} / C_{5}\right) \beta \lambda_{1, h_{k}}-\lambda_{2, h_{1}}<0$, then $\alpha_{1, k+1}=0$ and we have

$$
\begin{aligned}
\theta_{1, k+1}=\frac{\left|\bar{\lambda}_{1, h_{k+1}}-\alpha_{1, k+1}\right|}{\bar{\lambda}_{2, h_{k+1}}-\alpha_{1, k+1}} & =\frac{\left|\lambda_{1, h_{k}}+\left(\bar{\lambda}_{1, h_{k+1}}-\lambda_{1, h_{k}}\right)\right|}{\lambda_{2, h_{1}}+\bar{\lambda}_{2, h_{k+1}}-\lambda_{2, h_{1}}} \\
& =\frac{1}{2\left(1+C_{4} / C_{5}\right) \beta}+\mathcal{O}\left(h_{1}^{2}\right)
\end{aligned}
$$

Now, let us prove (19) by the method of induction. First, it is obvious that (19) holds for $n=1$. Then we assume that (19) holds for $n=k$. It means we have the following estimate:

$$
\left\|u_{1, h_{k}}-\Pi_{1, h_{k}} u_{1, h_{k}}\right\|_{a} \leq C_{5} \delta_{h_{k}}\left(\lambda_{1}\right) .
$$

Now let us consider the case of $n=k+1$. Combining (18), (25) and the triangle inequality leads to the following estimate:

$$
\begin{aligned}
\left\|u_{1, h_{k}}-\Pi_{1, h_{k+1}} u_{1, h_{k}}\right\|_{a} & \leq\left\|u_{1, h_{k}}-\Pi_{1, h_{k}} u_{1, h_{k}}\right\|_{a} \\
& +\left\|\Pi_{1, h_{k}} u_{1, h_{k}}-\Pi_{1, h_{k+1}} u_{1, h_{k}}\right\|_{a} \\
& \leq\left\|u_{1, h_{k}}-\Pi_{1, h_{k}} u_{1, h_{k}}\right\|_{a}+C_{4} \delta_{h_{k}}\left(\lambda_{1}\right) \\
& \leq C_{5}\left(1+\frac{C_{4}}{C_{5}}\right) \delta_{h_{k}}\left(\lambda_{1}\right) .
\end{aligned}
$$

From (12), (23), (24) and (26), we have

$$
\begin{aligned}
\frac{\theta_{1, k+1}}{1-\left(1+\theta_{1, k+1}\right)\left\|u_{1, h_{k}}-\Pi_{1, h_{k+1}} u_{1, h_{k}}\right\|_{a}} & =\frac{1}{2\left(1+C_{4} / C_{5}\right) \beta}+\mathcal{O}\left(h_{1}\right) \\
& <\frac{1}{\left(1+C_{4} / C_{5}\right) \beta},
\end{aligned}
$$

when $h_{1}$ is small enough.

From Theorem 3.3, (26) and (27), we have

$$
\left\|u_{1, h_{k+1}}-\Pi_{1, h_{k+1}} u_{1, h_{k+1}}\right\|_{a} \leq \frac{C_{5}}{\beta} \delta_{h_{k}}\left(\lambda_{1}\right)=C_{5} \delta_{h_{k+1}}\left(\lambda_{1}\right) .
$$


This means that the result (19) also holds for $n=k+1$. Thus we prove the desired result (19). From Lemma 3.1 and (19), we obtain the desired result (20). Finally, (22) follows from (19), (20) and the triangle inequality.

Remark 4.3 We also investigate the condition of the boundary value problem with different choices of $\alpha_{1, k+1}$. If we choose $\alpha_{1, k+1}$ as in (21) and $2\left(1+C_{4} / C_{5}\right) \beta \lambda_{1, h_{k}}-$ $\lambda_{2, h_{1}}>0$, then $\alpha_{1, k+1}<\bar{\lambda}_{1, h_{k+1}}$ when the mesh size $h_{1}$ is small enough. It means that (10) is a symmetric positive definite linear equation and its condition has the following estimate:

$$
\frac{\bar{\lambda}_{n, h_{k+1}}-\alpha_{1, k+1}}{\bar{\lambda}_{1, h_{k+1}}-\alpha_{1, k+1}} \approx \frac{\lambda_{1}}{\lambda_{1}-\alpha_{1, k+1}} \frac{\bar{\lambda}_{n, h_{k+1}}}{\bar{\lambda}_{1, h_{k+1}}} \approx \frac{2\left(1+C_{4} / C_{5}\right) \beta \lambda_{1}}{\lambda_{2}-\lambda_{1}} \operatorname{cond}(A),
$$

where $A$, cond $(A)$ and $\bar{\lambda}_{n, h_{k+1}}$ denote the stiff matrix, its condition number and the largest eigenvalue approximation of (6), respectively, in the finite element space $V_{h_{k+1}}$, the convergence results of $\lambda_{1, h_{k}}, \bar{\lambda}_{1, h_{k}}$ and $\lambda_{2, h_{1}}$ are used.

\section{Work estimate of eigenvalue multigrid scheme}

In this section, we turn our attention to the estimate of computational work for Algorithm 4.1. We will show that Algorithm 4.1 makes solving the eigenvalue problem need almost the same work as solving the corresponding boundary value problem if we adopt the multigrid method to solve the involved linear problem (10) $[4,7,8,19,20]$.

First, we define the dimension of each level linear finite element space as $N_{k}=$ $\operatorname{dim} V_{h_{k}}$. Then we have

$$
N_{k} \approx\left(\frac{1}{\beta}\right)^{d(n-k)} N_{n}, \quad k=1,2, \ldots, n .
$$

Theorem 5.1 Assume the eigenvalue problem solved in the coarse space $V_{h_{1}}$ needs work $\mathcal{O}\left(M_{h_{1}}\right)$ and the work of the multigrid solver in each level space $V_{h_{k}}$ is $\mathcal{O}\left(N_{k}\right)$ for $k=2,3, \ldots, n$. Then the total work involved in Algorithm 4.1 is $\mathcal{O}\left(N_{n}+M_{h_{1}}\right)$. Furthermore, the complexity will be $\mathcal{O}\left(N_{n}\right)$ provided $M_{h_{1}} \leq N_{n}$.

Proof Let $W_{k}$ denote the work of the correction step in the $k$-th finite element space $V_{h_{k}}$. Then with the correction definition, we have

$$
W_{k}=\mathcal{O}\left(N_{k}\right)
$$

Iterating (29) and using the fact (28), we obtain

$$
\begin{aligned}
\text { Total work } & =\sum_{k=1}^{n} W_{k}=\mathcal{O}\left(M_{h_{1}}+\sum_{k=2}^{n} N_{k}\right)=\mathcal{O}\left(M_{h_{1}}+\sum_{k=2}^{n} N_{k}\right) \\
& =\mathcal{O}\left(M_{h_{1}}+\sum_{k=2}^{n}\left(\frac{1}{\beta}\right)^{d(n-k)} N_{n}\right)=\mathcal{O}\left(N_{n}+M_{h_{1}}\right) .
\end{aligned}
$$


This is the desired result $\mathcal{O}\left(N_{n}+M_{h_{1}}\right)$ and the one $\mathcal{O}\left(N_{n}\right)$ can be obtained due to the condition $M_{h_{1}} \leq N_{n}$.

\section{Numerical results}

In this section, two numerical examples are presented to illustrate the efficiency of the multigrid scheme proposed in this paper.

\subsection{Model eigenvalue problem}

Here we give the numerical results of the multigrid scheme for the Laplace eigenvalue problem on the two-dimensional domain $\Omega=(0,1) \times(0,1)$. The sequence of finite element spaces is constructed by using linear element on the series of meshes which are produced by regular refinement with $\beta=2$ (producing $\beta^{d}$ congruent subelements). In this example, we use two meshes which are generated by the Delaunay method as the initial mesh $\mathcal{T}_{h_{1}}$ to produce two sequences of finite element spaces for investigating the convergence behaviors. Figure 1 shows the corresponding initial meshes: one is coarse and the other is fine.

Algorithm 4.1 is applied to solve the eigenvalue problem. For comparison, we also solve the eigenvalue problem by the direct method.

Figure 2 gives the corresponding numerical results for the first eigenvalue $\lambda_{1}=2 \pi^{2}$ and the corresponding eigenfunction on the two initial meshes illustrated in Fig. 1.

From Fig. 2, we find the multigrid scheme obtains the same optimal error estimates as the direct eigenvalue solving method for the eigenvalue and the corresponding eigenfunction approximations.

In order to show the efficiency of the method proposed in this paper, we also compare the running time of Algorithm 4.1 and the direct eigenvalue solving. Here we choose the package ARPACK $[10,11]$ as the direct eigenvalue solving tool to do the comparison. Here the conjugate gradient (CG) iteration and algebraic multigrid preconditioner are adopted to act as the linear solver in ARPACK. In Algorithm 4.1, we choose only the CG iteration as the linear solver. Both methods are running on the
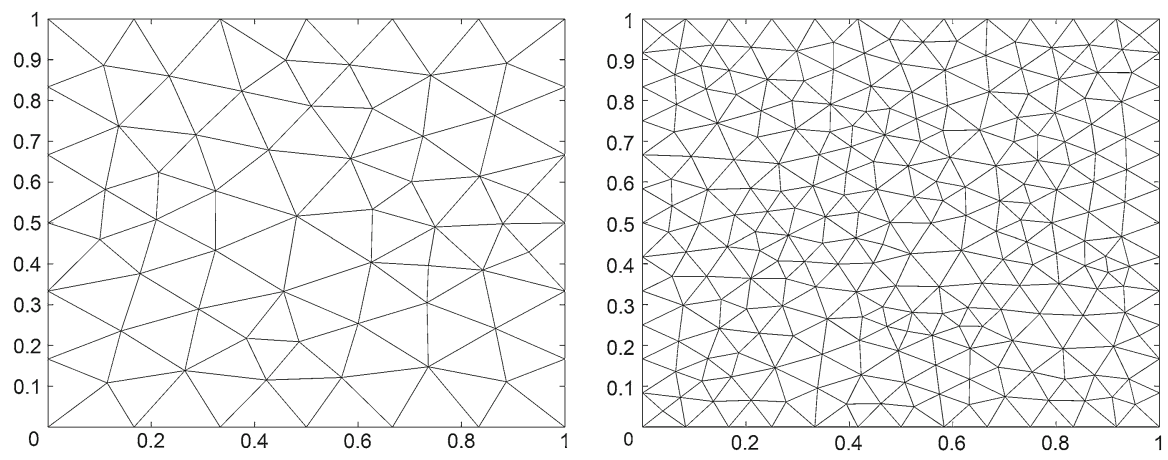

Fig. 1 The coarse and fine initial meshes for the first example 

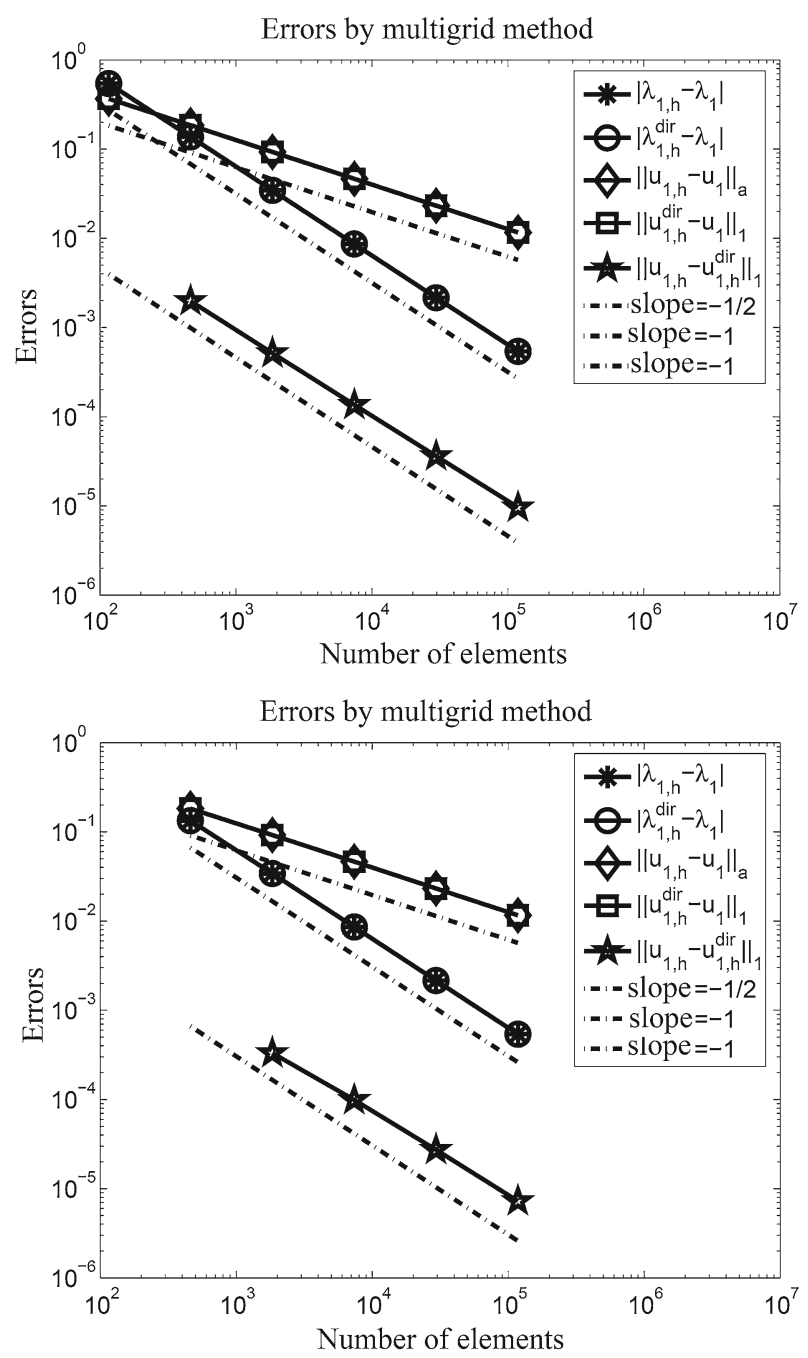

Fig. 2 The errors of the multigrid algorithm for the first eigenvalue $2 \pi^{2}$ and the corresponding eigenfunction, where $u_{h}^{\mathrm{dir}}$ and $\lambda_{h}^{\mathrm{dir}}$ denote the eigenfunction and eigenvalue approximation by direct eigenvalue solving (the first subfigure is for the coarse initial mesh in the first subfigure of Fig. 1 and the second one for the fine initial mesh in the second subfigure of Fig. 1)

same machine POWEREDGE R720 with the Linux system. The corresponding results are shown in Table 1 which implies that Algorithm 4.1 improves the efficiency of eigenvalue problem solving.

We also check the convergence behavior of multi eigenvalue approximations with Algorithm 4.1. Here the first six eigenvalues $\lambda=2 \pi^{2}, 5 \pi^{2}, 5 \pi^{2}, 8 \pi^{2}, 10 \pi^{2}, 10 \pi^{2}$ are investigated. We adopt the meshes in Fig. 1 as the initial meshes and the corresponding numerical results are shown in Fig. 3 which also exhibits the optimal convergence rate of the multigrid scheme. 
Table 1 The CPU time comparison between Algorithm 4.1 and direct eigenvalue solver. The number of elements in $V_{h_{1}}$ is 3,968

\begin{tabular}{lccc}
\hline Number of levels & Number of elements & Time for ARPACK & Time for Algorithm 4.1 \\
\hline 4 & 253,952 & 7.69 & 2.30 \\
5 & $1,015,808$ & 35.96 & 7.98 \\
6 & $4,063,232$ & 191.47 & 31.64 \\
7 & $16,252,928$ & $1,258.35$ & 127.08 \\
\hline
\end{tabular}
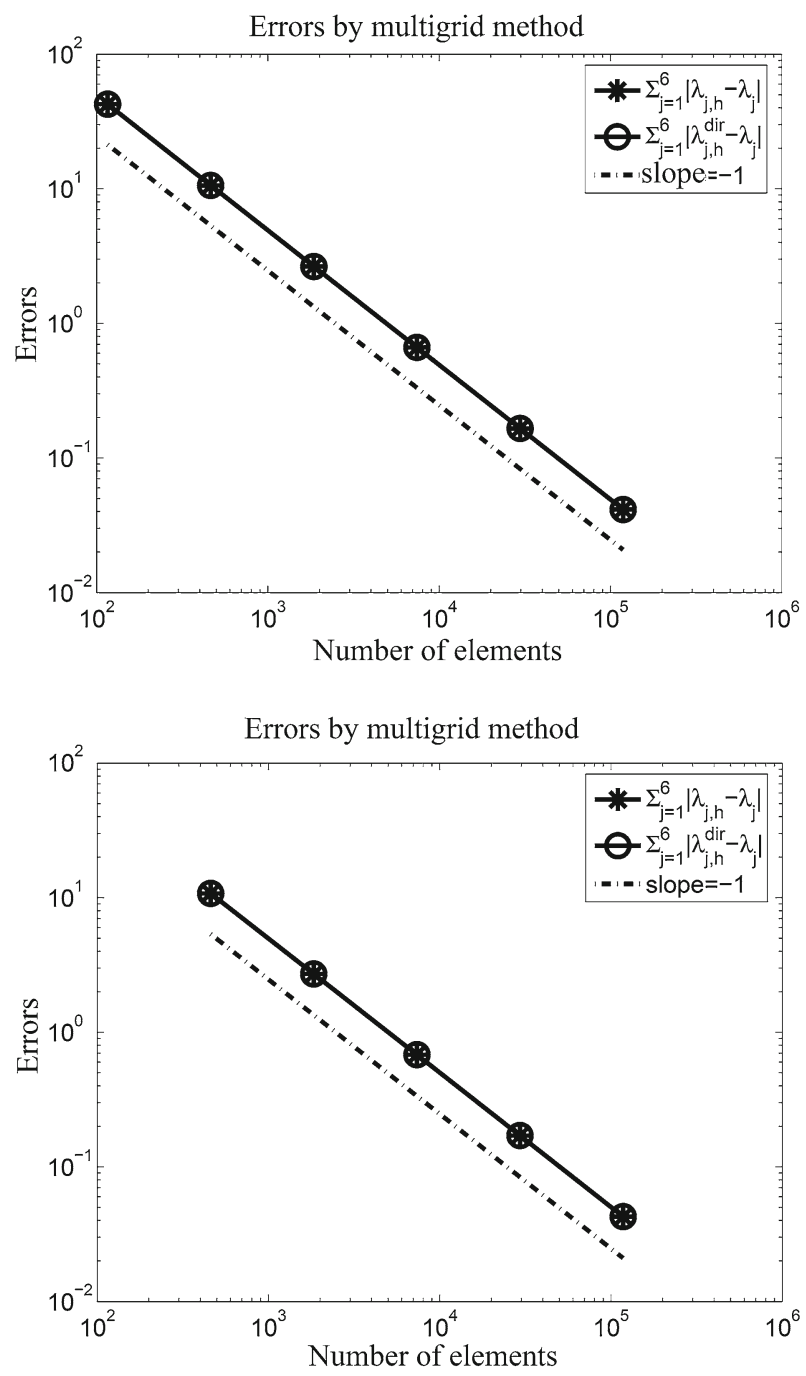

Fig. 3 The errors of the multigrid algorithm for the first six eigenvalues on the unit square (the first subfigure is for the coarse initial mesh in the first subfigure of Fig. 1 and the second one for the fine initial mesh in the second subfigure of Fig. 1) 
6.2 More general eigenvalue problem

Here we give the numerical results of the multigrid scheme for solving a more general eigenvalue problem on the unit square domain $\Omega=(0,1) \times(0,1)$ : Find $(\lambda, u)$ such that

$$
\begin{aligned}
-\nabla \cdot \mathcal{A} \nabla u+\phi u & =\lambda \rho u & & \text { in } \Omega, \\
u & =0 & & \text { on } \partial \Omega, \\
\int_{\Omega} \rho u^{2} d \Omega & =1, & &
\end{aligned}
$$

where

$$
\mathcal{A}=\left(\begin{array}{cc}
1+\left(x_{1}-1 / 2\right)^{2} & \left(x_{1}-1 / 2\right)\left(x_{2}-1 / 2\right) \\
\left(x_{1}-1 / 2\right)\left(x_{2}-1 / 2\right) & 1+\left(x_{2}-1 / 2\right)^{2}
\end{array}\right),
$$

$\phi=\mathrm{e}^{\left(x_{1}-1 / 2\right)\left(x_{2}-1 / 2\right)}$ and $\rho=1+\left(x_{1}-1 / 2\right)\left(x_{2}-1 / 2\right)$.

We first solve the eigenvalue problem (30) in the linear finite element space on the coarse mesh $\mathcal{T}_{h_{1}}$. Then refine the mesh by the regular way to produce a series of meshes $\mathcal{T}_{h_{k}}, k=2, \ldots, n$, with $\beta=2$ (connecting the midpoints of each edge) and solve the auxiliary boundary value problem (10) in the finer linear finite element space $V_{h_{k}}$ defined on $\mathcal{T}_{h_{k}}$.

In this example, we also use two coarse meshes which are shown in Fig. 1 as the initial meshes to investigate the convergence behaviors. Since the exact solution is not known, we choose an adequately accurate eigenvalue approximations with the extrapolation method [12] as the exact eigenvalue. Figure 4 gives the corresponding numerical results for the first six eigenvalue approximations and their corresponding eigenfunction approximations. Here we also compare the numerical results with the direct algorithm. Figure 4 also exhibits the optimal convergence rate of Algorithm 4.1.

\section{Concluding remarks}

In this paper, we give a type of multigrid scheme to solve eigenvalue problems. The idea here is to combine the shifted-inverse power iteration method with multilevel meshes to transform solving the eigenvalue problem to solving a series of the corresponding boundary value problems which can be done by the multigrid method. As stated in the numerical examples, EIGENVAluE MulTIGRID SCHEME defined in Algorithm 4.1 for one eigenvalue can be extended to the corresponding version for multi eigenvalues (including simple and multiple eigenvalues). We state the following version of EIGENVALUE MULTIGRID SCHEME for $m$ eigenvalues.

Similarly, we first give a type of ONE CORRECTION STEP FOR Multi EIGENVALUES for the given eigenpairs approximations $\left\{\lambda_{j, h_{k}}, u_{j, h_{k}}\right\}_{j=1}^{m}$.

\section{Algorithm 7.1 One CORRECTION STEP FOR Multi EIGENVAlues}

1. Do $j=1, \ldots, m$

- Find $\tilde{u}_{j, h_{k+1}} \in V_{h_{k+1}}$ such that for all $v_{h_{k+1}} \in V_{h_{k+1}}$,

$$
a\left(\tilde{u}_{j, h_{k+1}}, v_{h_{k+1}}\right)-\alpha_{j, k+1} b\left(\widetilde{u}_{j, h_{k+1}}, v_{h_{k+1}}\right)=b\left(u_{j, h_{k}}, v_{h_{k+1}}\right) .
$$



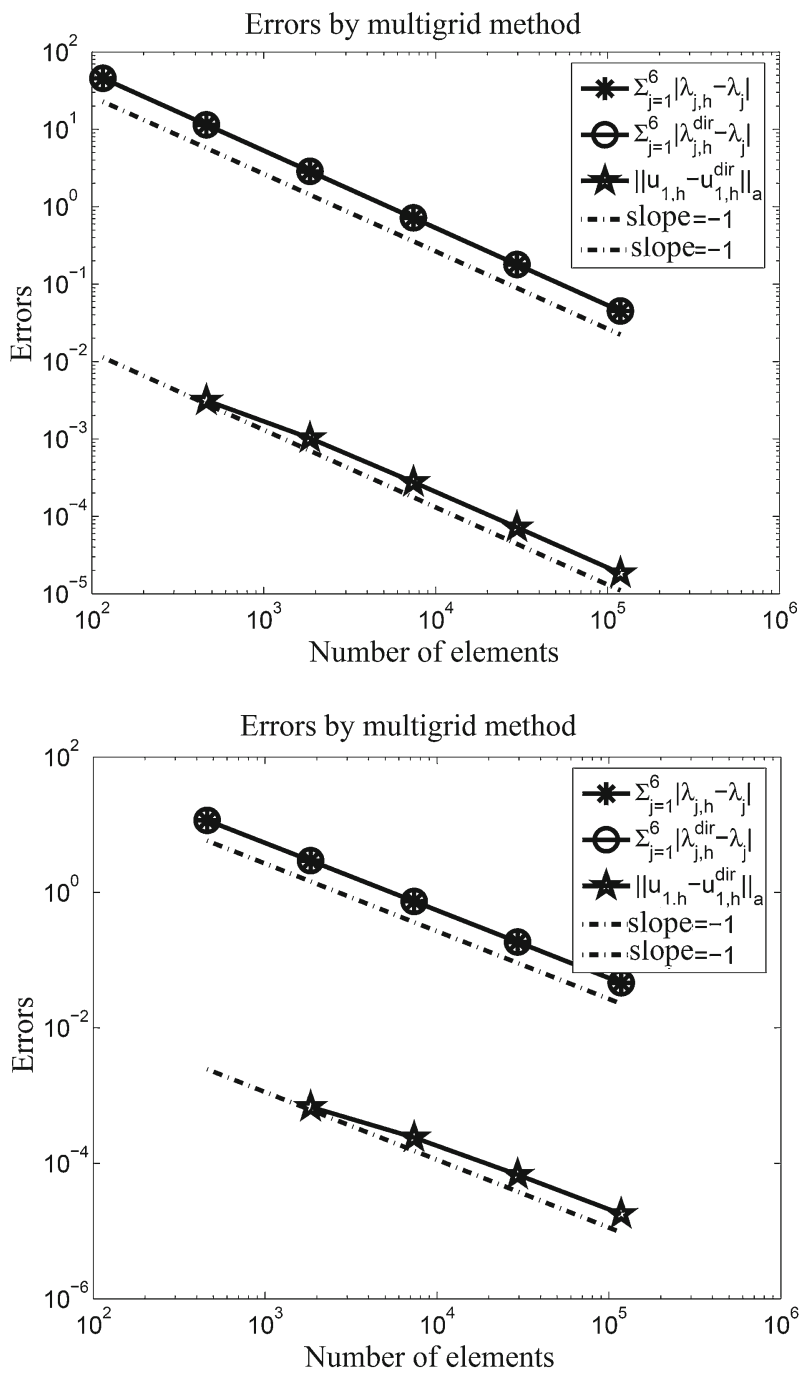

Fig. 4 The errors of the multigrid algorithm for the first six eigenvalues and the first eigenfunction, where $u_{h}^{\text {dir }}$ and $\lambda_{h}^{\text {dir }}$ denote the eigenfunction and eigenvalue approximation by direct eigenvalue solving (the first subfigure is for the coarse initial mesh in the first subfigure of Fig. 1 and the second one for the fine initial mesh in the second subfigure of Fig. 1)

- Do the following orthogonalization and normalization:

$$
\begin{aligned}
\widehat{u}_{j, h_{k+1}} & =\widetilde{u}_{j, h_{k+1}}-\sum_{\ell=1}^{j-1} a\left(\widetilde{u}_{j, h_{k+1}}, u_{\ell, h_{k+1}}\right) u_{\ell, h_{k+1}}, \\
u_{j, h_{k+1}} & =\frac{\widehat{u}_{j, h_{k+1}}}{\left\|\widehat{u}_{j, h_{k+1}}\right\|_{a}} .
\end{aligned}
$$

\section{End Do}


2. Compute the new eigenvalue approximations

$$
\lambda_{j, h_{k+1}}=\frac{a\left(u_{j, h_{k+1}}, u_{j, h_{k+1}}\right)}{b\left(u_{j, h_{k+1}}, u_{j, h_{k+1}}\right)}, \quad j=1, \ldots, m .
$$

We summarize the above two steps into

$$
\left\{\lambda_{j, h_{k+1}}, u_{j, h_{k+1}}\right\}_{j=1}^{m}=\operatorname{Correction}\left(\left\{\alpha_{j, k+1}\right\}_{j=1}^{m},\left\{\lambda_{j, h_{k}}, u_{j, h_{k}}\right\}_{j=1}^{m}, V_{h_{k+1}}\right) .
$$

Similarly to (3.5), we can also define a modified one correction step for multi eigenvalues where we need to solve a small dimensional eigenvalue problem.

\section{Algorithm 7.2 One CORRECTION STEP FOR Multi Eigenvalues}

1. Do $j=1, \ldots, m$

Find $\widetilde{u}_{j, h_{k+1}} \in V_{h_{k+1}}$ such that for all $v_{h_{k+1}} \in V_{h_{k+1}}$,

$$
a\left(\widetilde{u}_{j, h_{k+1}}, v_{h_{k+1}}\right)-\alpha_{j, k+1} b\left(\widetilde{u}_{j, h_{k+1}}, v_{h_{k+1}}\right)=b\left(u_{j, h_{k}}, v_{h_{k+1}}\right) .
$$

\section{End Do}

2. Build a finite dimensional space $\widetilde{V}_{h_{k+1}}=\operatorname{span}\left\{\widetilde{u}_{1, h_{k+1}}, \ldots, \widetilde{u}_{m, h_{k+1}}\right\}$ and solve the following eigenvalue problem: Find $\left(\lambda_{j, h_{k+1}}, u_{j, h_{k+1}}\right) \in \mathbb{R} \times \widetilde{V}_{h_{k+1}}, j=$ $1,2, \ldots, m$, such that $a\left(u_{j, h_{k+1}}, u_{j, h_{k+1}}\right)=1$ and

$$
a\left(u_{j, h_{k+1}}, v_{h_{k+1}}\right)=\lambda_{j, h_{k+1}} b\left(u_{j, h_{k+1}}, v_{h_{k+1}}\right), \quad v_{h_{k+1}} \in \widetilde{V}_{h_{k+1}} .
$$

We summarize the above two steps into

$$
\left\{\lambda_{j, h_{k+1}}, u_{j, h_{k+1}}\right\}_{j=1}^{m}=\operatorname{Correction}\left(\left\{\alpha_{j, k+1}\right\}_{j=1}^{m},\left\{\lambda_{j, h_{k}}, u_{j, h_{k}}\right\}_{j=1}^{m}, V_{h_{k+1}}\right) \text {. }
$$

Based on Algorithm 7.1 or 7.2, we can give the corresponding multigrid method.

Algorithm 7.3 Eigenvalue Multigrid Scheme for Multi Eigenvalues

1. Construct a series of nested finite element spaces $V_{h_{1}}, V_{h_{2}}, \ldots, V_{h_{n}}$ such that (16) and (17) hold.

2. Solve the following eigenvalue problem: Find $\left(\lambda_{h_{1}}, u_{h_{1}}\right) \in \mathbb{R} \times V_{h_{1}}$ such that $a\left(u_{h_{1}}, u_{h_{1}}\right)=1$ and

$$
a\left(u_{h_{1}}, v_{h_{1}}\right)=\lambda_{h_{1}} b\left(u_{h_{1}}, v_{h_{1}}\right), \quad v_{h_{1}} \in V_{h_{1}} .
$$

Choose $m$ eigenpairs $\left\{\lambda_{j, h_{1}}, u_{j, h_{1}}\right\}_{j=1}^{m}$ which approximate our desired eigenvalues and their eigenspaces.

3. Do $k=1, \ldots, n-1$

Obtain new eigenpair approximations $\left\{\lambda_{j, h_{k+1}}, u_{j, h_{k+1}}\right\}_{j=1}^{m} \in \mathbb{R} \times V_{h_{k+1}}$ by a correction step

$$
\left\{\lambda_{j, h_{k+1}}, u_{j, h_{k+1}}\right\}_{j=1}^{m}=\text { Correction }\left(\left\{\alpha_{j, k+1}\right\}_{j=1}^{m},\left\{\lambda_{j, h_{k}}, u_{j, h_{k}}\right\}_{j=1}^{m}, V_{h_{k+1}}\right) .
$$

\section{End Do}


Finally, we obtain $m$ eigenpair approximations $\left\{\lambda_{j, h_{n}}, u_{j, h_{n}}\right\}_{j=1}^{m} \in \mathbb{R} \times V_{h_{n}}$.

We can also define

$$
\alpha_{j, k+1}=\max \left\{0, \frac{2\left(1+C_{4} / C_{5}\right) \beta \lambda_{j, h_{k}}-\lambda_{j+1, h_{k}}}{2\left(1+C_{4} / C_{5}\right) \beta-1}\right\}, \quad j=1, \ldots, m-1,
$$

and

$$
\alpha_{m, k+1}=\max \left\{0, \frac{2\left(1+C_{4} / C_{5}\right) \beta \lambda_{m, h_{k}}-\lambda_{m+1, h_{1}}}{2\left(1+C_{4} / C_{5}\right) \beta-1}\right\} .
$$

Based on the above definitions of $\alpha_{j, k+1}$, we can also give the error analysis for this version of eigenvalue multigrid method in the similar way as in Sects. 3 and 4. If we use the mulgirid method (the multigrid method for indefinite problems from $[15,20]$ ) to solve the boundary value problems included in Algorithm 7.3, the computational work involved in the multi eigenvalues version is $\mathcal{O}\left(m^{2} N_{n}\right)$. Furthermore, the parallel computation can be used to solve (31) and (32) for different $j$. The analysis of the scheme for multi eigenvalues will be given in our future work.

We can replace the multigrid method by other types of efficient iteration schemes such as algebraic multigrid method, the type of preconditioned schemes based on the subspace decomposition and subspace corrections [4,19], and the domain decomposition method [16]. Furthermore, the framework here can also be coupled with the parallel method and the adaptive refinement technique. The ideas should be extended to other types of linear eigenvalue problems. These will be investigated in our future work.

\section{References}

1. Babuška, I., Osborn, J.E.: Finite element-Galerkin approximation of the eigenvalues and eigenvectors of selfadjoint problems. Math. Comp. 52(186), 275-297 (1989)

2. Babuška, I., Osborn, J.: Eigenvalue problems. In: Ciarlet, P.G., Lions, J.L. (eds.) Handbook of Numerical Analysis, Vol. II. Finite Element Methods, Part 1, pp. 641-787. North-Holland, Amsterdam (1991)

3. Brandt, A., McCormick, S., Ruge, J.: Multigrid methods for differential eigenproblems. SIAM J. Sci. Stat. Comput. 4(2), 244-260 (1983)

4. Brenner, S.C., Scott, L.R.: The Mathematical Theory of Finite Element Methods. Texts in Applied Mathematics, vol. 15. Springer, New York (1994)

5. Chatelin, F.: Spectral Approximation of Linear Operators. Computer Science and Applied Mathematics. Academic Press, New York (1983)

6. Ciarlet, P.G.: The Finite Element Method for Elliptic Problems. Studies in Mathematics and its Applications, vol. 4. North-Holland, Amsterdam (1978)

7. Hackbusch, W.: On the computation of approximate eigenvalues and eigenfunctions of elliptic operators by means of a multi-grid method. SIAM J. Numer. Anal. 16(2), 201-215 (1979)

8. Hackbusch, W.: Multi-Grid Methods and Applications. Springer Series in Computational Mathematics, vol. 4. Springer, Berlin (1985)

9. Hu, X., Cheng, X.: Acceleration of a two-grid method for eigenvalue problems. Math. Comp. 80(275), 1287-1301 (2011)

10. Lehoucq, R., Maschhoff, K., Sorensen, D., Yang, C.: ARPACK Software Package (1996). http://www. caam.rice.edu/software/ARPACK/

11. Lehoucq, R.B., Sorensen, D.C., Yang, C.: ARPACK Users' Guide. Software, Environments, and Tools, vol. 6. SIAM, Philadelphia (1998)

12. Lin, Q., Lin, J.: Finite Element Methods. China Science and Technology Press, Beijing (2005) 
13. Lin, Q., Xie, H.: A multi-level correction scheme for eigenvalue problems. Math. Comp. 84(291), 71-88 (2015)

14. Lin, Q., Yan, N.: The Construction and Analysis of High Efficiency Finite Element Methods. Hebei University Press, Hebei (1995) (in Chinese)

15. Shaidurov, V.V.: Multigrid Methods for Finite Element. Mathematics and its Applications, vol. 318. Kluwer, Dordrecht (1995)

16. Toselli, A., Widlund, O.: Domain Decomposition Methods-Algorithms and Theory. Springer Series in Computational Mathematics, vol. 34. Springer, Berlin (2005)

17. Xie, H.: A type of multilevel method for the Steklov eigenvalue problem. IMA J. Numer. Anal. 34(2), 592-608 (2014)

18. Xie, H.: A multigrid method for eigenvalue problem. J. Comput. Phys. 274, 550-561 (2014)

19. Xu, J.: Iterative methods by space decomposition and subspace correction. SIAM Rev. 34(4), 581-613 (1992)

20. Xu, J.: A new class of iterative methods for nonselfadjoint or indefinite problems. SIAM J. Numer. Anal. 29(2), 303-319 (1992)

21. Xu, J., Zhou, A.: A two-grid discretization scheme for eigenvalue problems. Math. Comp. 70(233), 17-25 (2001)

22. Yang, Y., Bi, H.: Two-grid finite element discretization schemes based on shifted-inverse power method for elliptic eigenvalue problems. SIAM J. Numer. Anal. 49(4), 1602-1624 (2011) 